\title{
Educación a distancia ante la emergencia sanitaria por COVID-19. Condiciones, procesos y perspectivas de estudiantes normalistas
}

\section{Distance education in the face of the health emergency for COVID-19. Conditions, processes and students' perceptions in the Normal}

\author{
BRITO-LARA, Maribel*†, HERRERA-RENDÓN, Enrique, PATRÓN-REYES, Armida Liliana y \\ TERÁN-MONTENEGRO, Alfonso de la Luz
}

Escuela Normal Superior Oficial de Guanajuato. Guanajuato, México

ID $1^{\text {er }}$ Autor: Maribel, Brito-Lara / ORC ID: 0000-0002-2800-3436

ID 1 ${ }^{\mathrm{er}}$ Autor: Enrique, Herrera-Rendón / ORC ID: 0000-0001-5585-1791

ID $2^{\text {do }}$ Autor: Armida Liliana, Patrón-Reyes / ORC ID: 0000-0002-8024-3568

ID $3^{\text {er }}$ Autor: Alfonso de la Luz, Terán-Montenegro / ORC ID: 0000-0001-9087-6345

DOI: $10.35429 / \mathrm{JCP} .2020 .12 .4 .6 .14$

Recibido 16 de Agosto, 2020; Aceptado 30 de Diciembre, 202

Resumen

Se presentan resultados de un estudio empírico mixto con diseño transversal, de tipo descriptivo; cuya finalidad es conocer las condiciones, procesos y percepciones estudiantiles respecto de la educación a distancia con el uso de medios digitales llevada a cabo ante la situación de confinamiento por la pandemia de COVID-19. Participaron 208 estudiantes de licenciaturas para la formación de docentes de secundaria. Se empleó un cuestionario con preguntas abiertas y de opción múltiple. Con base en los hallazgos se advierte que la brecha digital está presente de manera importante; los educandos, "nativos digitales", presentan oportunidad de mejora en cuanto al empleo de medios digitales con fines de estudio y trabajo. El cambio a modalidad a distancia influyó, predominantemente, de forma adversa; si bien en varios casos representó oportunidad para el desarrollo personal y profesional. La experiencia de trabajo a distancia es mayormente valorada como "regular" (40.5\%) y "buena" (33\%), y la atención docente ante situaciones particulares que incidían en el desempeño estudiantil como "buena" (53\%) y "regular" (22\%). Se plantean, como propuestas principales para la mejora: a) de parte de los docentes más y mejor comunicación con los discentes, compromiso y organización del trabajo, así como sensibilidad para comprender las situaciones que rebasan las posibilidades estudiantiles; b) a nivel institucional, capacitación en el uso de medios digitales para docentes y estudiantes, y unificación del tipo de plataforma de trabajo. Los compromisos estudiantiles se concentran en mayor disposición y responsabilidad ante el trabajo.

Educación a distancia, Medios digitales, Estudiantes normalistas, COVID-19

\begin{abstract}
This article presents the results of a mixed empirical study with a cross-sectional design, of a descriptive type, the purpose of which is to know the conditions, processes and student perceptions regarding distance education with the use of digital media carried out in the situation of confinement due to the pandemic of COVID-19. 208 undergraduate students in several majors in high school teaching participated. A questionnaire with open-ended and multiple-choice questions was used. Based on the findings, it is noted that the digital divide is present in an important way; the learners that are "digital natives" present opportunities for improvement in the use of digital media for study and work purposes. The shift to distance mode had a predominantly adverse effect; however, in several cases, it represented an opportunity for personal and professional development. The distance work experience is mostly valued as "not great" (40.5\%) and "good" $(33 \%)$, and the attention given to particular situations that affected student achievement as "good" (53\%) and "not great" $(22 \%)$. The article's main proposals for improvement are: a) on the part of teachers more and better communication with the learners, commitment and organization of work, as well as sensitivity to understand situations that exceed student possibilities; b) at the institutional level, training in the use of digital media for teachers and students, and unification of the type of work platform. Student commitments focus on greater enthusiasm and responsibility for work.
\end{abstract}

Distance education, Digital media, Normal students, COVID-19

Citación: BRITO-LARA, Maribel, HERRERA-RENDÓN, Enrique, PATRÓN-REYES, Armida Liliana y TERÁNMONTENEGRO, Alfonso de la Luz. Educación a distancia ante la emergencia sanitaria por COVID-19. Condiciones, procesos y perspectivas de estudiantes normalistas. Revista de Pedagogía Crítica. 2020, 4-12: 6-14

\footnotetext{
* Correspondencia al Autor (Correo electrónico: mabila68@gmail.com)

$\dagger$ Investigador contribuyendo como primer autor.
} 


\section{Introducción}

Transcurría el ciclo escolar 2019-2020, en la Escuela Normal Superior Oficial de Guanajuato (ENSOG), los procesos de enseñanza y aprendizaje para la formación inicial de docentes de secundaria se desarrollaban de acuerdo con lo planeado por cada uno de los profesores; pero desde abril 2020, por la oficial medida de confinamiento para contrarrestar la epidemia generada por el virus SARS-CoV2 -COVID-19, se pasó del trabajo presencial en las aulas a la modalidad educativa a distancia. Si bien el empleo de medios digitales se había venido incorporando en los procesos educativos, ahora su uso se convertía en una emergente necesidad; situación para la que la comunidad educativa no estaba preparada, pero fue necesario atender buscando alcanzar las metas de aprendizaje previstas.

Posicionándonos en la perspectiva de los estudiantes normalistas, mayores usuarios y beneficiarios de los servicios que ofrece la citada institución educativa, nos preguntamos: ¿qué condiciones para la educación a distancia con el uso de medios digitales presenta tal población?, ¿cómo valoran su desempeño académico ante tal modalidad de trabajo?, ¿qué estrategias estudiantiles fueron las más empleadas?, ¿de qué manera la situación derivada de la contingencia sanitaria y la medida de confinamiento influyó en su desempeño académico?, ¿cómo valoran la atención recibida por parte de sus profesores y; en general, tal experiencia de trabajo?, ¿qué propuestas planten para mejorar el desarrollo de los cursos en dicha modalidad?, ¿qué compromisos asumirían, ante una necesidad similar u oportunidad posterior? Con base en estas cuestiones, se busca tener un acercamiento a las condiciones, procesos y percepciones estudiantiles respecto de la educación a distancia con el uso de medios digitales, desarrollado ante la medida de confinamiento por la pandemia de COVID-19.

\section{Metodología}

\section{Tipo de Estudio y Participantes}

Se llevó a cabo un estudio empírico mixto. Es descriptivo, ya que se busca caracterizar la situación y participación de los estudiantes en las clases a distancia con el empleo de recursos digitales (Vargas, 2012).
Participaron 208 estudiantes (66.5\% de la matrícula) de los diversos programas de Licenciatura para la formación de docentes de secundaria que se ofrecen en la ENSOG, sede Guanajuato. Al final del ciclo escolar, se envió invitación a toda la población estudiantil (312), a través de correo electrónico, explicándoles la finalidad de la encuesta y reiterándoles el uso confidencial que se haría de sus respuestas.

\section{Instrumento y Análisis de la Información}

Se empleó una encuesta con preguntas de opción múltiple y preguntas abiertas; la cual fue revisada y ajustada con base en observaciones hechas por cuatro colegas docentes e investigadores. Del primer tipo de preguntas: cinco fueron para caracterizar a la población de estudio, quince para explorar acerca de la disposición de recursos digitales y habilitación para su uso; quince para autoevaluación del desempeño y determinación de estrategias de trabajo empleadas y, una para valoración de la experiencia educativa en modalidad a distancia con el empleo de recursos digitales. El total de preguntas abiertas fueron cuatro, con lo cual se exploró: la influencia del confinamiento por la contingencia sanitaria en el desempeño académico, la valoración de la atención docente recibida, las propuestas estudiantiles para mejorar el desarrollo de los cursos mediante la citada modalidad y, los compromisos que asumirían ante una necesidad similar $u$ oportunidad posterior de trabajo. La encuesta fue construida y aplicada con la herramienta de Google Forms.

Para el análisis de la información obtenida mediante las preguntas de opción múltiple, se calculó la frecuencia mediante la aplicación Excel de Microsoft Office. Las respuestas de las preguntas abiertas se agruparon en categorías que "concentran las ideas, conceptos o temas similares descubiertos" (Fernández N., 2006).

\section{Resultados}

\section{Características de los participantes}

Con base en los datos obtenidos, cuyo análisis se presenta en la tabla 1, de los 208 participantes, predominan los del sexo femenino (66\%); asimismo quienes se forman para ejercer la docencia en la modalidad de telesecundaria $(33 \%)$.

BRITO-LARA, Maribel, HERRERA-RENDÓN, Enrique, PATRÓNREYES, Armida Liliana y TERÁN-MONTENEGRO, Alfonso de la Luz. Educación a distancia ante la emergencia sanitaria por COVID-19. Condiciones, procesos y perspectivas de estudiantes normalistas. Revista de Pedagogía Crítica. 2020 
No hay gran discrepancia entre la proporción de quienes cursan uno u otro plan de estudios en operación (Plan 1999: 49\% y Plan 2018: 51\%). El $87 \%$ de los normalistas pertenecen a dos rangos de edad: 20-22 y 17-19 años, lo que indica que han cursado sus estudios dentro del tiempo ordinario previsto en la normatividad escolar. Si bien hay predominio de quienes proceden del medio urbano (44\%); los del medio rural representan una proporción importante que se ubica en segundo lugar (35\%). La mitad de los estudiantes atienden también otras actividades; predominantemente se desempeñan como empleados (34\%), en varios casos porque sus familiares se quedaron sin empleo debido a la contingencia sanitaria.

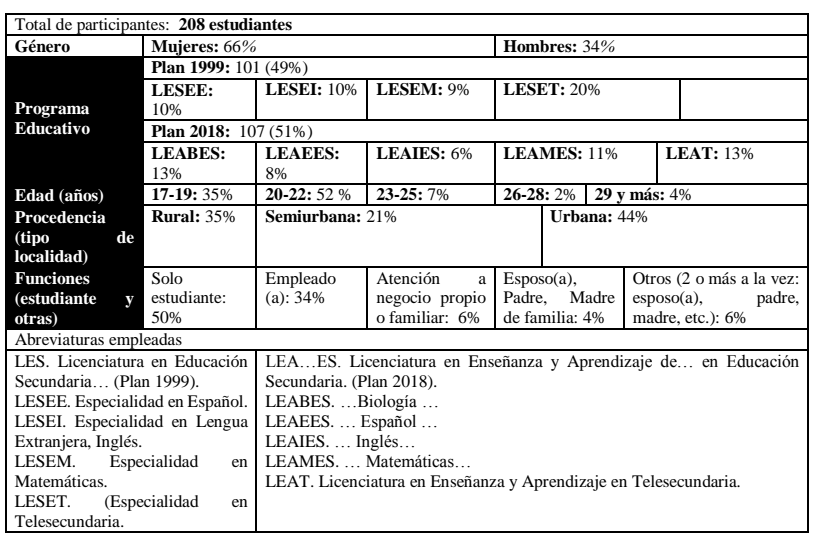

Tabla 1 Características de la población participante Fuente: Elaboración Propia

\section{Condiciones para el trabajo estudiantil a distancia con el uso de medios digitales}

1. Disponibilidad de dispositivos digitales y conectividad a internet.

Con base en lo que se señala en la tabla 2, a pesar de que la gran mayoría de estudiantes (93\%) dispone de smartphone, poco más de dos tercios de ellos (69\%) regularmente no tiene datos en sus dispositivos. La mitad de los participantes comparten su equipo de cómputo, casi una cuarta parte entre tres o más usuarios. Es amplia la mayoría que dispone de internet en casa; pero predomina una regular capacidad y velocidad del servicio, que en conjunto con un mal servicio alcanza el $75 \%$.

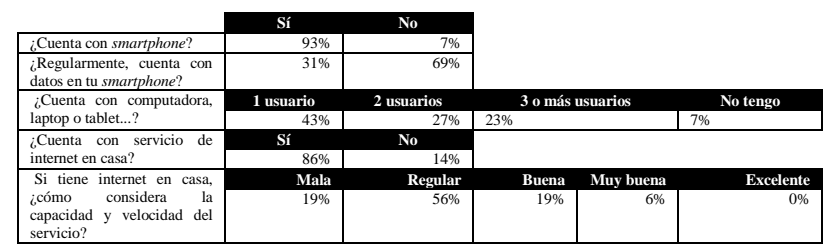

Tabla 2 Disponibilidad de dispositivos digitales y conectividad a internet

Fuente: Elaboración Propia

ISSN 2523-2479

ECORFAN $^{\circledR}$ Todos los derechos reservados
2. Nivel de dominio en el manejo de medios digitales

Con base en lo señalado en la tabla 3, predominan los niveles "bueno" y "excelente". Los mayores niveles de dominio en el manejo de medios digitales corresponden al uso de redes sociales y chats, así como de internet y correo electrónico. En cuanto al empleo de plataformas digitales, los niveles "bueno" y "excelente", alcanzan el 65\%. Llama la atención que un tercio de la población presenta "regular" nivel de dominio en herramientas de Office, Plataformas digitales, así como Blogs y foros de discusión, medios que esencialmente son empleados para fines de trabajo o estudio.

\begin{tabular}{|l|r|r|r|r|r|}
\hline \multicolumn{1}{l|}{} & Nulo & Incipiente & Regular & Bueno & Excelente \\
$\begin{array}{l}\text { Oerramientas de } \\
\text { Office: Word, } \\
\text { Excel y Power } \\
\text { point }\end{array}$ & & $4 \%$ & $33 \%$ & $52 \%$ & $11 \%$ \\
\hline $\begin{array}{l}\text { Internet y correo } \\
\text { electrónico }\end{array}$ & $0 \%$ & $2 \%$ & $21 \%$ & $52 \%$ & $25 \%$ \\
\hline $\begin{array}{l}\text { Redes sociales y } \\
\text { chats }\end{array}$ & $0 \%$ & $3 \%$ & $17 \%$ & $53 \%$ & $27 \%$ \\
\hline $\begin{array}{l}\text { Blogs y foros de } \\
\text { discusión }\end{array}$ & $3 \%$ & $13 \%$ & $37 \%$ & $44 \%$ & $4 \%$ \\
\hline $\begin{array}{l}\text { Plataformas } \\
\text { digitales; } \\
\text { Classroom, } \\
\text { Edmodo u otras }\end{array}$ & $0 \%$ & $4 \%$ & $31 \%$ & $50 \%$ & $15 \%$ \\
\hline
\end{tabular}

Tabla 3 Nivel de dominio en el manejo de medios digitales

Fuente: Elaboración Propia

3. Maneras para habilitarse o mejorar habilidades en el uso de medios digitales

Múltiples fueron las maneras que emplearon los estudiantes normalistas para su habilitación en el uso de los medios digitales; como se señala en la tabla 4 , predomina la consulta con sus compañeros de clase, seguida de la consulta de manuales o tutoriales.

\begin{tabular}{|c|c|c|}
\hline & Sí & No \\
\hline Consulté a compañeros del grupo. & $83 \%$ & $17 \%$ \\
\hline Consulté a familiares y amigos. & $61 \%$ & $39 \%$ \\
\hline Consulté manuales o tutoriales. & $76 \%$ & $24 \%$ \\
\hline Participé en cursos, webinars, etc. & $20 \%$ & $80 \%$ \\
\hline Otras & $33 \%$ & $67 \%$ \\
\hline
\end{tabular}

Tabla 4 Maneras para habilitación en el uso de medios digitales

Fuente: Elaboración propia
BRITO-LARA, Maribel, HERRERA-RENDÓN, Enrique, PATRÓNREYES, Armida Liliana y TERÁN-MONTENEGRO, Alfonso de la Luz. Educación a distancia ante la emergencia sanitaria por COVID-19. Condiciones, procesos y perspectivas de estudiantes normalistas. Revista de Pedagogía Crítica. 2020 
II.Los estudiantes ante la educación a distancia con el uso de medios digitales

1. Desempeño académico y estrategias de trabajo

La autovaloración del desempeño académico (Tabla 5) indica que, en lo general, predominaron los desempeños "bueno" y "excelente"; destacan la atención a las instrucciones de trabajo, seguido de la atención a los criterios de evaluación al realizar sus producciones, la revisión y adecuación de sus producciones antes de entregarlas para revisión de sus profesores y, el desarrollo de competencias del curso o asignatura. La mayor proporción en los niveles "regular", "básico" e "incipiente" se presenta en la facilidad para trabajar de forma autónoma; seguido de la organización de tiempos y dedicación suficiente para las actividades según su complejidad, así como en la facilidad para manejar herramientas digitales.

\begin{tabular}{|l|r|r|r|r|r|}
\hline \multicolumn{1}{|c|}{ Descriptores Incipiente } & \multicolumn{1}{|c|}{ Básico } & Regular & Bueno & Excelente \\
\hline $\begin{array}{l}\text { Organicé mis tiempos y } \\
\text { dediqué lo suficiente } \\
\text { para las actividades } \\
\text { según su complejidad. }\end{array}$ & $3 \%$ & $8 \%$ & $19 \%$ & $41 \%$ & $29 \%$ \\
\hline $\begin{array}{l}\text { Atendí las instrucciones } \\
\text { de trabajo. }\end{array}$ & $1 \%$ & $4 \%$ & $8 \%$ & $55 \%$ & $32 \%$ \\
\hline $\begin{array}{l}\text { Atendí los criterios de } \\
\text { evaluación al realizar } \\
\text { las producciones } \\
\text { (trabajos). }\end{array}$ & $0 \%$ & $5 \%$ & $10 \%$ & $53 \%$ & $32 \%$ \\
\hline $\begin{array}{l}\text { Se me facilitó trabajar } \\
\text { de forma autónoma. }\end{array}$ & $4 \%$ & $9 \%$ & $22 \%$ & $38 \%$ & $26 \%$ \\
\hline $\begin{array}{l}\text { Entregué mis trabajos } \\
\text { oportunamente. }\end{array}$ & $4 \%$ & $6 \%$ & $17 \%$ & $31 \%$ & $41 \%$ \\
\hline $\begin{array}{l}\text { Me fue fácil manejar } \\
\text { herramientas digitales } \\
\text { (plataformas, } \\
\text { documentos } \\
\text { colaborativos, etc.). }\end{array}$ & $3 \%$ & $8 \%$ & $18 \%$ & $42 \%$ & $29 \%$ \\
\hline $\begin{array}{l}\text { Aproveché las TIC para } \\
\text { potenciar } \\
\text { aprendizajes. mis }\end{array}$ & $2 \%$ & $9 \%$ & $11 \%$ & $47 \%$ & $32 \%$ \\
\hline $\begin{array}{l}\text { En actividades en } \\
\text { equipo, participé } \\
\text { activamente y aporté lo } \\
\text { que me correspondió. }\end{array}$ & $1 \%$ & $5 \%$ & $13 \%$ & $32 \%$ & $49 \%$ \\
\hline $\begin{array}{l}\text { Me integré, sin } \\
\text { inconvenientes, en } \\
\text { equipos organizados } \\
\text { con criterios diferentes } \\
\text { a a la afinidad entre } \\
\text { compañeros. }\end{array}$ & $2 \%$ & $7 \%$ & $12 \%$ & $38 \%$ & $41 \%$ \\
\hline $\begin{array}{l}\text { Revisé y adecué mis } \\
\text { producciones antes de } \\
\text { entregarlas dos para } \\
\text { revisión de los } \\
\text { profesores. }\end{array}$ & $0 \%$ & $4 \%$ & $12 \%$ & $43 \%$ & $41 \%$ \\
\hline $\begin{array}{l}\text { Desarrollé } \\
\text { competencias previstas } \\
\text { en el curso o asignatura. }\end{array}$ & & & & & \\
\hline
\end{tabular}

Tabla 5 Desempeño académico estudiantil Fuente: Elaboración Propia
Respecto de las estrategias para el trabajo (Tabla 6); esforzarse por realizar más adecuadamente las actividades de profesores que consideran más exigentes tuvo predomino, pues "casi siempre" y "siempre" trabajaban en tal sentido; en segundo lugar, cumplir primero con actividades de la línea de acercamiento a la práctica escolar (LAPE) y el trayecto de práctica profesional.

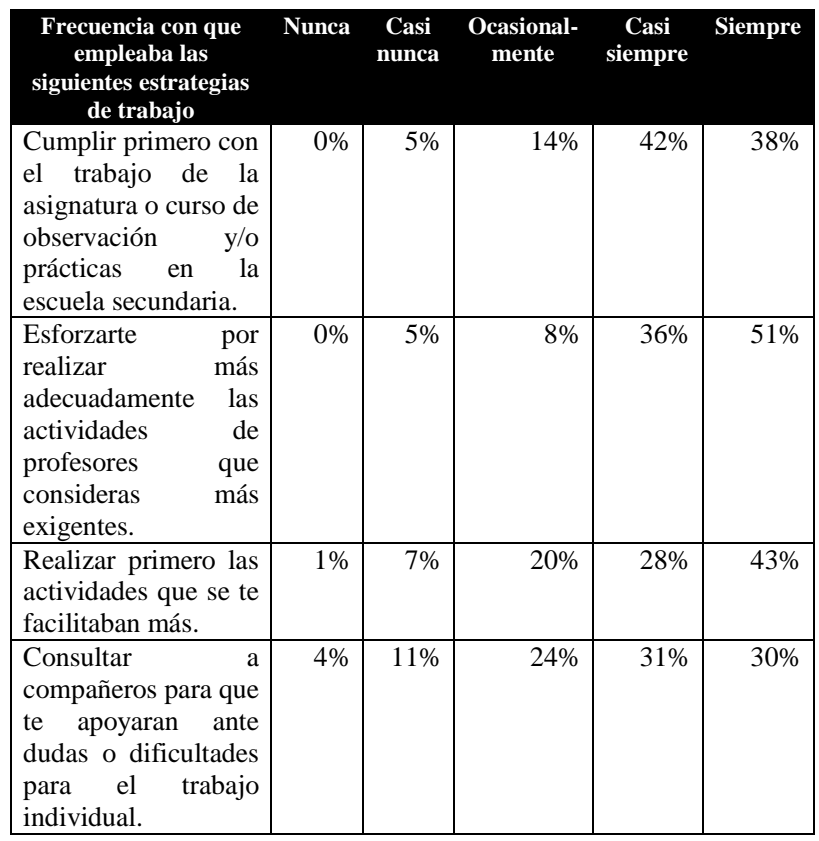

Tabla 6 Estrategias estudiantiles para el trabajo (frecuencia de empleo)

Fuente: Elaboración Propia

2. Influencia de la contingencia sanitaria y medida de confinamiento en el desempeño académico estudiantil

Predominantemente, se alude influencia adversa:

a. En el rendimiento escolar y en lo socioemocional (18\%): “-Afecta en la comprensión de instrucciones, en la elaboración de los trabajos. Los trabajos eran extensos y no entendibles". "-Poco aprendizaje o poco significativos". "-Baja en los promedios de calificación, afectación en rendimiento escolar". Dificultad para alcanzar el nivel de entendimiento que se creía tener antes de esta situación. "-Generó mucho estrés". En algunos casos, familiares o conocidos se contagiaron (algunos siguen delicados) y eso afectó la atención y/o aprendizaje. “-Desmotivación por la modalidad de trabajo (sin interacción directa con compañeros, docentes y la escuela en general), se trabajaba, pero al final, más por obligación que por convicción..." Generó ansiedad, incertidumbre.

BRITO-LARA, Maribel, HERRERA-RENDÓN, Enrique, PATRÓNREYES, Armida Liliana y TERÁN-MONTENEGRO, Alfonso de la Luz. Educación a distancia ante la emergencia sanitaria por COVID-19. Condiciones, procesos y perspectivas de estudiantes normalistas. Revista de Pedagogía Crítica. 2020 
Problemas de concentración y de desempeño en el trabajo académico.

b. Contrastó con la preferencia y estilos de aprendizaje (17\%). “-Evitó que existiera comunicación bilateral fluida para aclarar las actividades a realizar, las dudas que surgen". "Faltó acompañamiento y retroalimentación de los maestros". Dificultades para organizar tiempos para atender las diversas actividades, para trabajar de forma autónoma. Sentimiento de soledad que generó el aislamiento; "-no compartir e interactuar con compañeros de manera presencial, fue algo difícil de asimilar".

c. La tecnología fue un problema $(9 \%)$. Complicaciones (económicas, tiempo, riesgo de contagio) por tener que salir al ciber. Dificultades en el uso de medios tecnológicos, eso hacía que se complicaran las actividades académicas y se invirtiera más tiempo en realizarlo. "-En algunas materias (de contenidos disciplinarios, p. ej.) se requiere más consulta en internet o explicación docente...". "-No se tiene internet en casa o el servicio que se tiene no es bueno...". No se tiene o tenía computadora o se descompuso.

d. Exceso de actividades (8\%). Aumento en carga de actividades en casa (atender a hermanos menores, ayudar en actividades domésticas, asesoría tecnológica, etc.). "-Cargas de trabajo escolar (mucho tiempo en la computadora...)". Tener que trabajar para ayudar a pagar los gastos familiares (familias de bajos recursos o padres se quedaron sin empleo).

e. Hizo falta la práctica (4\%). “-El no haber completado la fase práctica dejó algunos conocimientos teóricos incompletos. Se vio afectada la realización del DR". "-Esperaba que mi preparación fuera aún más completa, empaparme de los elementos necesarios para concluir con el ciclo escolar en curso, mi preocupación era llegar a mi último año de licenciatura con dudas sobre el trabajo a desarrollar en 7mo y 8vo semestre, así como forjar una estrategia en la práctica docente que me favoreciera para el análisis en mi DR.”. “-No aprender en la cuestión práctica dejando los aprendizajes teóricos sin bases". f. Otras $(17 \%)$. Algunos profesores pidieron materiales o pidieron realizar una actividad fuera de casa". "-... los maestros no quieren tomar su papel, y tenemos que hacer nuestras cosas de manera autónoma". "-Las clases se volvieron más teóricas", las actividades de práctica profesional en las escuelas secundarias fueron suspendidas. "-La verdad faltó mucha retroalimentación por parte de los maestros, en ningún momento me enteré si la mayoría de los trabajos estaban bien o el porqué de su calificación".

Pero también hubo respuestas $(27 \%)$ en el sentido de que la experiencia sirvió de aprendizaje:

a. Ayudó al crecimiento personal y autonomía. "-Generó reflexión, crecimiento personal, ser responsable". Ayudó para aprender a adaptarse a los cambios, estudiar/aprender con autonomía. "-Permitió aprender a organizarse y manejar los tiempos para hacer los trabajos y tareas, aunque al principio fueron demasiados en algunas materias después hubo un equilibrio". "Permitió darse cuenta de las propias debilidades y capacidades; así como de los retos y las posibilidades que se dan en el ámbito académico".

b. Permitió fortalecer habilidades en el uso de Tecnologías de la Información y la Comunicación (TIC): “- $-\ldots$ aprender a trabajar con una nueva modalidad (plataformas y otros medios digitales) a la que no se estaba acostumbrado. Esta experiencia también servirá para el ejercicio como docente de Secundaria". Aprendizajes en cuanto a comunicación mediante las herramientas electrónicas e incluso a dirigir clases online. "-Aprendizajes sobre el uso de nuevas plataformas digitales para la creación de trabajos como libros digitales, infografías, etc." "-A hacer un mejor uso de las tecnologías para investigar y seguir aprendiendo".

3. Valoración de la atención recibida por parte de sus profesores y; en general, de la experiencia educativa a distancia

129 estudiantes expresaron haber solicitado algún tipo de atención particular a sus profesores, debido a situaciones personales que enfrentaron por la situación de contingencia sanitaria y la medida de confinamiento. 
Sus valoraciones a la atención recibida y comentarios típicos que expresan son los siguientes:

a. Excelente, 12\%." "-Todos mis profesores estuvieron muy al pendiente de todas las situaciones por las que atravesamos en esta contingencia". "-Los profesores mostraron empatía frente a las situaciones planteadas". "Excelente nunca me dejaron solo solo [SIC] hubo una maestra que no me apoto [SIC] y pues lamentablemente reprobé". "-De Maravilla, ayudaron con mi ansiedad y me escucharon". "Excelente, pues se me brindo [SIC] el apoyo y la experiencia"

b. Muy buena, 11\%. "- $-\ldots$ siempre estaban con toda la disposición de atender a mis dudas". "-Con los que tuve ese acercamiento se portaron muy bien."

c. Buena, 53\%. "-Considero que fue buena y flexible". "-Buena, aunque algunos maestros no comprendieron las situaciones presentadas".

d. Regular, 22\%. " $-\ldots$ pues no todos respondieron y los que lo hicieron no fue a tiempo". "-... había algunos maestros que tardaban todo un día para responder a las dudas que surgían. $Y$ a veces sus explicaciones no eran del todo claras. "-... a veces no respondían algunos docentes". "-7 de 10, apenas y pasan"

e. Mala, 2\%. Las valoraciones que dan a la experiencia de trabajo a distancia con el empleo de medios digitales es la siguiente: a) Excelente, .5\%; b) Muy buena, $13 \%$; c) Buena, $33 \%$; d) Regular, $40.5 \%$; e) Mala, $13 \%$.

4. Propuestas para mejorar el desarrollo de los cursos en modalidad a distancia.

Las propuestas, abarcan tres sentidos:

a. Lo que directamente corresponde a la planta docente $(71 \%)$.
Más y mejor comunicación de profesores con estudiantes. Que comuniquen los criterios de trabajo al inicio del curso, den instrucciones claras y precisas, así como retroalimentación; que las instrucciones se den en tiempo real en línea y den la clase virtual; “-...algunos docentes (que no lo han estado) estén disponibles para resolver dudas, porque mandan trabajo y no sabes de ellos hasta él siguiente trabajo"; "-Principalmente, que los docentes estén en constante comunicación con los grupos, tengan la atención, disposición y conciencia que requerimos para cumplir en tiempo y forma con lo que se nos solicita"; se generen grupos de WhatsApp y otras formas de comunicación más directa. Que las actividades sean de manera individual, ya que para realizar tareas en equipo es difícil porque no todos tienen la misma disposición.

- Compromiso y organización del trabajo. Actualización de materiales proporcionados por los profesores, así como la organización entre los mismos para distribuir los trabajos que se piden a fin de que no se junten. "-Hay maestros que caen en la falta de motivación y de dedicación, cayendo en cumplir sólo por cubrir los temas, sin asegurarse que haya comprensión por parte de los estudiantes", que las actividades sean dinámicas, y se evite el trabajo excesivo y repetitivo.

- Comprensión. Se solicita que los maestros sean tolerantes y comprensivos ante las situaciones particulares de los estudiantes, puesto que es frecuente que no se tenga siempre internet, que no siempre se esté en casa, o que se tenga que ir a trabajar. "-Empatía y comprensión por parte de maestros (no todos)."

b. Los que la institución educativa puede impulsar (24\%).

Respecto del uso de la tecnología. Trabajar todas las asignaturas con un solo correo y en un mismo tipo de plataforma digital; que se den más herramientas de apoyo y se den capacitaciones para maestros y alumnos. 
- $\quad$ Respecto del trabajo docente y horarios de trabajo. Establecer políticas para que los maestros den a conocer con anticipación los trabajos que se realizarán por parcial; dar capacitación a los docentes para que puedan dar clases virtuales; trabajar sólo en horas de clase y evitar dejar trabajo los fines de semana; trabajar un mes y una semana descanso para que los estudiantes despejen su mente de pasar la mayoría del día realizando actividades y; que no se quite del todo el trabajo presencial.

Por otra parte, 5\% expresan agradecimiento y reconocimiento al trabajo docente (atención, orientación, retroalimentación) y aluden la necesidad del compromiso conjunto entre profesores y estudiantes.

5. Compromisos que asumirían ante una posterior necesidad $\mathrm{u}$ oportunidad similar de trabajo

a. Mayor responsabilidad y disposición es lo que más señalan (71\%). Entrega adecuada y oportuna y de los trabajos, "-Estar al pendiente de las indicaciones de igual manera cumplir con los trabajos y estar en las clases virtuales", "-Apoyar a los compañeros", mantener el rendimiento académico, mejorar las áreas de oportunidad, dedicarse, atender y participar más en las clases.

b. $17 \%$ refieren sobre la mejor organización del tiempo y destinar el necesario para las actividades académicas.

c. $8 \%$ aluden al desarrollo de nuevas habilidades y mejoramiento de las que ya se tienen para el empleo de las TIC, así como destinar recursos para disponer de servicio de internet.

d. $4 \%$ expresan lo relacionado con mejorar la comunicación entre compañeros, así como entre estudiantes y docente; de modo que se disipen dudas respecto de lo abordado en clase y de atiendan conflictos que puedan presentarse.

\section{Discusión}

La brecha digital es una condición importante en la modalidad educativa emergente. Si bien los participantes, que mayoritariamente proceden de poblaciones rurales y semiurbanas, superan la proporción media estatal de usuarios (estudiantes de licenciatura) de equipo de cómputo, internet y telefonía móvil: $22.8 \%$, $18.1 \%$ y $15.2 \%$, respectivamente (Rojas y Navarrete, 2019); la mitad de ellos comparten su equipo de cómputo con una o más personas; solo una tercera parte de quienes tienen smartphone (93\%) regularmente cuenta con datos en sus dispositivos y; quienes disponen de servicio de internet en casa $(86 \%)$, la calidad de este es mayoritariamente $(75 \%)$ de "regular" a "mala".

Los estudiantes, "nativos digitales", presentan oportunidad de mejora importante en cuanto a habilidades para el empleo de medios digitales con fines de estudio y trabajo; pues como se advierte, una tercera parte de ellos señalan tener un nivel de dominio "regular" en cuanto al uso de blogs y foros de discusión, herramientas de Office y plataformas.

En cuanto a los procesos y perspectivas de los estudiantes ante lo que se ha denominado educación a distancia de emergencia, el cambio de modalidad tuvo influencia predominantemente adversa. Destacan aspectos socioemocionales como desmotivación, estrés, ansiedad e incertidumbre; en varios casos familiares o amigos enfermaron de COVID-19; su rendimiento escolar presentó descenso, se les dificultó comprender instrucciones para las actividades $\mathrm{y}$ estas les representaron poco aprendizaje o poca significatividad. Echaron de menos la relación física cercana con compañeros y docentes, se sentían solos, se les dificultó el trabajo autónomo, no lograban organizar sus tiempos de modo que pudieran atender sus cargas de actividades (académicas y no); en muchos casos tuvieron también fallas o indisponibilidad de recursos digitales, así como complicaciones para emplearlos en el trabajo académico con efectividad y facilidad. 
Las adversidades referidas, con base en lo que señala UNESCO a través del Instituto Internacional para la Educación Superior en América Latina y el Caribe -IESALC-, (2020), pueden entenderse como resultado de que el diseño curricular no está concebido en modalidad a distancia; no hubo preparación previa (docente ni estudiantil); las expectativas de desarrollo de competencias docentes están puesta en el trabajo presencial con actividades prácticas en el futuro campo laboral; asimismo la citada modalidad educativa requiere de mayor disciplina y compromiso estudiantil.

A pesar de la preeminente percepción adversa, una cuarta parte de los estudiantes señalan que la experiencia de trabajo a distancia les representó oportunidad para su crecimiento personal y profesional; de fortalecimiento para el trabajo autónomo y el desarrollo de habilidades para el uso de TIC; este tipo de percepciones representa autoconfianza, categoría que forma parte de las trayectorias vitales que definen a los buenos profesores (López, 2010), y es que justamente la formación inicial representa un escenario en que se construyen y ponen en juego saberes para el futuro ejercicio profesional.

La "buena" a "excelente" atención que los educandos recibieron de sus profesores $(76 \%$ en conjunto), ante situaciones particulares que enfrentaron el proceso formativo debido a la contingencia sanitaria y la medida de confinamiento, da cuenta de la mística de la planta docente, a pesar de las afectaciones de tipo laboral y profesionales que el profesorado pueda estar enfrentando (UNESCO-IESALC, 2020); aunado a las de tipo personal. En un sentido un tanto contrastante, la valoración general que los estudiantes dan a la experiencia del trabajo a distancia con el uso de medios digitales, muestra predominio en el nivel "regular" (40.5\%) y en conjunto con la valoración en el nivel "malo" comprende el $53.5 \%$. En ambos casos hay oportunidad de mejora, para lo cual las propuestas y compromisos estudiantiles son un referente. Lo que proponen los estudiantes para mejorar el desarrollo de los cursos en la modalidad de trabajo emergente; en primer lugar, corresponde a lo que ellos consideran habrían de ser atendidas directamente por los docentes: más y mejor comunicación con los estudiantes, compromiso y organización del trabajo, así como sensibilidad para comprender las situaciones que rebasan las posibilidades de los educandos.
En segundo lugar, sus propuestas están dirigidas a atención institucional, entre ellas: capacitación a docentes y estudiantes en el uso de recursos digitales y, unificación de tipo de plataforma para el trabajo. Los compromisos que señalan asumirían se centran en mayor disposición y responsabilidad ante el trabajo.

Los hallazgos representan un insumo valioso para la toma de decisiones orientada a la formación de profesionales de la educación en y para la complejidad e incertidumbre imperantes en nuestra era; si bien es necesario recurrir a la mirada de los demás miembros de la comunidad educativa. Los medios digitales no son un paliativo ante una situación emergente, representan una herramienta valiosísima para la construcción de una sociedad del conocimiento (Flores R., Aguilar B., Hernández P., Salazar T., \& Pinillos V., 2017), aspiración que convoca a estar abiertos a aprender y aprovechar las coyunturas que podemos encontrar, incluso en las adversidades.

\section{Referencias}

de Maturana Luna, S. L. (2010). Historia de vida de buenos profesores: experiencia e impacto en las aulas. Profesorado, revista de currículum y formación del profesorado,14(3), 149-164. https://www.redalyc.org/pdf/567/56715702011. pdf

Fernández N., L. (2006). ¿Cómo analizar datos cualitativos? Butletí LaRecerca. https://ebevidencia.com/wpcontent/uploads/2014/12/analisis-datoscualitativos.pdf

Flores R., M., Aguilar B., A. J., Hernández P., Y. K., Salazar T., J. p., \& Pinillos V., J. A. (2017). Sociedad del conocimiento, las TIC y su influencia en la educación. Espacios, 38(35), 3951.

https://www.revistaespacios.com/a17v38n35/a1 7v38n35p39.pdf

Rojas M., I. y Navarrete C., Z. (2019). Modalidades no presenciales de educación superior en México: composición, tendencias y desafios. México: Universidad Nacional Autónoma de México. Facultad de Filosofía y Letras.

http://ru.atheneadigital.filos.unam.mx/jspui/han dle/FFYL_UNAM/408 
UNESCO IESALC (2020). Covid-19 y educación superior: de los efectos inmediatos al día después. Análisis de impactos, respuesta y recomendaciones. París, Francia: UNESCO. http://www.iesalc.unesco.org/wpcontent/uploads/2020/04/COVID-19-060420ES-2.pdf

Varga B., X. (2012). ¿Cómo hacer investigación cualitativa? $2^{\mathrm{a}}$. Edición. México: Etxeta. 